Relations industrielles

Industrial Relations

\title{
Deux systèmes de relations industrielles en Belgique (textile et fabrications métalliques), par Thérèse Klein-Beaupain, Bruxelles, Éditions de l’Université de Bruxelles, 1979, 335 pp.
}

\section{Jean Boivin}

Volume 35, numéro 3, 1980

URI : https://id.erudit.org/iderudit/029102ar

DOI : https://doi.org/10.7202/029102ar

Aller au sommaire du numéro

Éditeur(s)

Département des relations industrielles de l'Université Laval

ISSN

0034-379X (imprimé)

1703-8138 (numérique)

Découvrir la revue

Citer ce compte rendu

Boivin, J. (1980). Compte rendu de [Deux systèmes de relations industrielles en Belgique (textile et fabrications métalliques), par Thérèse Klein-Beaupain, Bruxelles, Éditions de l'Université de Bruxelles, 1979, 335 pp.] Relations industrielles / Industrial Relations, 35(3), 596-598.

https://doi.org/10.7202/029102ar

Tous droits réservés @ Département des relations industrielles de l'Université Laval, 1980
Ce document est protégé par la loi sur le droit d'auteur. L'utilisation des services d’Érudit (y compris la reproduction) est assujettie à sa politique d'utilisation que vous pouvez consulter en ligne.

https://apropos.erudit.org/fr/usagers/politique-dutilisation/ 


\section{RECENSIONS BOOK REVIEWS}

Deux systèmes de relations industrielles en Belgique (textile et fabrications métalliques), par Thérèse Klein-Beaupain, Bruxelles, Éditions de l'Université de Bruxelles, 1979, 335 pp.

Cet ouvrage constitue l'une des rares applications au contexte européen de l'approche anglo-saxonne et nord-américaine pour analyser les relations industrielles. En effet, l'auteur étudie la situation des négociations et des conventions collectives dans deux industries belges - le textile et les fabrications métalliques - à partir des concepts développés par les grands spécialistes tels John T. Dunlop (E.U.), Allan Flanders (G.B.), R. Dahrendorf (G.B.), A. Fox (G.B.), R. Dubin (E.U.), N. Chamberlain (E.U.) et H. Clegg (G.B.), pour ne nommer que les plus importants.

Une telle étude fait contraste avec la plupart des études européennes qui ont l'habitude d'analyser les relations industrielles de façon globale et à partir de schèmes idéologiques bien particuliers. L'auteur ayant acquis une maîtrise en sociologie de l'Université Columbia (New York), on y retrouve sans doute là l'explication de l'approche originale qu'elle développe. Car au delà des contributions britanniques et américaines mentionnées plus haut, cet ouvrage, qui constitue un extrait de la thèse de doctorat de l'auteur, puise aussi abondamment dans la littérature du continent européen. C'est ainsi que les contributions de François Sellier, Yves Delamotte, Roger Blanpain, Daniel Vidal et Dimitri Weiss sont largement mises à partie.

Le volume comprend quatre parties distinctes: la première fixe le cadre général d'analyse et c'est sans doute celle qui consti- tue l'apport le plus substantiel pour le lecteur intéressé par la théorie de la négociation collective et l'étude des systèmes de relations industrielles. On y retrouve une excellente revue de la littérature sur les aspects aussi fondamentaux que les sources du conflit industriel et sur les caractéristiques essentielles de la négociation collective, considérée comme mécanisme de participation à la prise de décision ainsi que comme processus d'élaboration de règles. Cette première partie permet aussi de situer les contextes économiques et institutionnels dans lesquels se déroulent les négociations collectives en plus de présenter les principaux acteurs impliqués, notamment les fédérations syndicales et les associations patronales. Enfin, un dernier chapitre effectue un survol de l'ensemble des accords survenus au niveau inter-professionnel (c'est-à-dire de l'ensemble de l'économie) au cours de la période étudiée, à savoir 1960 à 1975. Ces accords, même s'ils ne font pas à proprement parler partie de l'étude, constituent néanmoins une toile de fond susceptible de conditionner les négociations au niveau des secteurs industriels.

La deuxième partie est consacrée à l'évolution de la négociation collective dans chacune des deux industries de 1960 à 1975 .

Même si le sujet porte sur l'étude de la négociation collective, le lecteur nordaméricain éprouve une certaine difficulté à comprendre la signification réelle de ce processus dans le contexte européen. La principale différence provient du caractère très centralisé de ces négociations, ce qui fait contraste avec la situation que l'on connaît ici. En effet, non seulement s'agit-il de véritables négociations sectorielles (quoique l'auteur souli- 
gne l'existence d'accords régionaux et d'entreprise), mais de plus, ces négociations s'effectuent souvent dans le prolongement «d'accords inter-professionnels de programmation sociale», sortes de sommets économiques qui déterminent des conditions minimales applicables à l'ensemble des agents socioéconomiques.

Une deuxième différence provient de l'absence quasi totale d'encadrement juridique à l'intérieur duquel se déroulent les négociations. Les parties n'ont pas à respecter des procédures strictes régissant le droit de grève et, très souvent, des accords sont conclus avant même l'expiration des accords précédents. D'ailleurs, dans l'industrie textile du moins, on retrouve toute une gamme d'accords tels les accords généraux, les accords de programmation sociale, les accords intérimaires et les accords supplémentaires. De telles négociations présentent donc les apparences d'une véritable négociation continue, puisque dans le secteur du textile on dénombre pas moins de vingt accords survenus dans la période 1960-1975, alors qu'il y en a eu dix dans le secteur des fabrications métalliques, secteur où les relations entre les parties sont qualifiées de «beaucoup moins cordiales» que dans l'industrie textile.

La troisième partie du volume analyse les différents objets ou résultats de négociations, en distinguant quatre sujets principaux: les salaires, la durée du travail, les fonds de sécurité d'existence (ou avantages complémentaires de sécurité sociale) et les relations collectives, en respectant encore une fois la dichotomie «industrie textile» et «fabrications métalliques».

Il est intéressant d'apprendre qu'à cause de la plus grande centralisation des décisions dans l'industrie du textile, les augmentations de salaires sont négociées sectoriellement et applicables à toutes les entreprises alors que dans l'industrie de fabrications métalliques, cette question est renvoyée aux régions et aux entreprises individuelles. C'est pourquoi, dans cette dernière industrie, les entreprises plus prospères dépassent les augmentations prévues dans les accords nationaux, ce qui n'est pas le cas dans l'industrie textile.
D'autre part, au cours de la période étudiée, les modifications apportées à la durée hebdomadaire du travail (réduction) et aux vacances annuelles (allongement) ont provoqué une diminution d'environ $15 \%$ du nombre d'heures productives par travailleur et par an dans les deux industries.

L'existence de «fonds de sécurité d'existence» financés par les cotisations des seuls employeurs et prévoyant le versement de diverses prestations aux travailleurs, telles des suppléments aux allocations de chômage versées par le régime universel, des compléments de pension ou d'allocations familiales, un pécule complémentaire de vacances, etc., ne mériterait pas que l'on souligne le sujet puisque nos conventions collectives nordaméricaines contiennent elles aussi un ensemble de clauses prévoyant divers bénéfices sociaux, si ce n'était de certaines caractéristiques originales. En effet, ces fonds prévoient aussi des avantages aux seuls travailleurs syndiqués, ce qui serait illégal selon notre législation du travail, ainsi que certains versements aux organisations syndicales.

De tels fonds dans chacune des deux industries furent le résultat de revendications syndicales maintes fois répétées. Cependant, lorsque les employeurs acceptèrent de consentir à procurer des avantages aux seuls syndiqués, ils obtinrent en contrepartie des clauses de "paix sociale» plus ou moins contraignantes pour les syndicats et en vertu desquelles ceux-ci acceptèrent de limiter leur liberté de revendiquer. Enfin, la liaison entre la paix sociale et le versement d'une prime syndicale financée par les employeurs est assortie d'un système de sanctions. Ainsi, dans l'industrie textile, c'est le respect de la paix sociale qui conditionne les versements effectués par le fonds. Malgré tout, l'auteur souligne que les sanctions ne procurent pas une assurance totale contre les conflits comme l'indiquent les statistiques concernant les jours perdus à cause des grèves; quoique l'existence de telles clauses de paix sociale permet, à tout le moins, de mieux réglementer le conflit.

Dans la quatrième partie, intitulée «conclusions générales», l'auteur essaie de relier les constatations des deux parties précédentes 
aux considérations théoriques de la première partie. Elle en arrive à formuler des propositions qui confirment des réalités vécues en Amérique du Nord, comme par exemple, «le fait qu'en échange de la reconnaissance totale du fait syndical, les organisations de travailleurs se sont retrouvées liées par des engagements très contraignants en matière de paix sociale et de procédure de conciliation") ( $p$. 256). Ou encore, "qu'une organisation devenue significative au niveau de la société globale tend à servir les intérêts de l'organisation comme telle (sa continuité et son expansion matérielle), au lieu de demeurer le moyen de réaliser les finalités pour lesquelles elle s'est créée» (p. 257).

L'étude de Thérèse Klein-Beaupain corrobore également certains résultats d'autres recherches, notamment celles réalisées en Grande-Bretagne par Clegg et Flanders, et qui révèlent que plus les règles de fond d'une convention collective sectorielle sont souples, plus les règles de procédures ont tendance à être développées et contraignantes.

L'auteur fait également des observations intéressantes, sur la distribution des tâches qui s'est opérée entre les quatre niveaux de négociations que l'on retrouve en Belgique, à savoir: le niveau inter-professionnel, le niveau professionnel (sectoriel), le niveau régional professionnel et le niveau de l'entreprise. Cependant, le lecteur nord-américain peut difficilement en saisir toute la pertinence étant donné la place prédominante occupée par la négociation d'entreprise sur ce continent.

Il ne fait aucun doute que le volume Deux systèmes de relations industrielles en Belgique constitue un apport important à la recherche en relations industrielles dans la mesure où la méthode comparative utilisée par l'auteur est l'une des plus valables pour expliquer le fonctionnement des systèmes de relations industrielles.

Jean BOIVIN

Université Laval
Les syndicats indépendants au Québec: un aperçu de leur situation, par François Delorme et Diane Veilleux, Coll. «Etudes et recherches", Centre de recherche et de statistiques sur le marché du travail, Québec, ministère du Travail et de la Main-d'oeuvre, 1980, 95 pp.

Il y a près de vingt ans, paraissait aux États-Unis une étude America's Forgotten Labor Organization (Princeton, 1962). L'auteur, Arthur B. Shostak, mettait en lumière l'importance, la nature, le rôle et le fonctionnement de ces groupements dont les journaux font rarement mention et qui n'ont pas non plus donné lieu à des recherches systématiques de la part des observateurs des problèmes de relations du travail. Nous avions alors fait une recension de cet ouvrage dans Relations industrielles (vol. 18, 1963, no 1).

Les syndicats indépendants du Québec: un aperçu de leur situation vient donc combler une lacune chez nous. C'est la première fois qu'un tel sujet y est abordé d'une façon systématique. L'étude est divisée en quatre chapitres: éléments de la problématique; l'identification des syndicats inclépendants; quelques facettes de l'action syndicale menée par les syndicats indépendants et perspectives et avenues de recherches. Le tout est suivi d'une excellente bibliographie.

Deux problèmes majeurs se sont posés aux auteurs avant d'entreprendre leur inventaire et leur analyse. D'abord, celui de déterminer ce que l'on entend par syndicat indépendant et ensuite trouver les sources d'information pour les repérer.

La notion du syndicat indépendant est difficilement notion opérationnelle. Une chose est claire: un syndicat indépendant n'est pas nécessairement un syndicat jaune ou un syndicat dominé par l'employeur. Mais il est indépendant parce que non rattaché à une centrale syndicale. Cela repose la question à savoir ce que recouvre la notion de centrale syndicale. Nous reconnaissons que la définition que nous avons présentée dans notre Dictionnaire canadien des relations du travail mériterait d'être plus élaborée pour devenir à son tour opérationnelle. Le sens commun, la 